\title{
USO INADEQUADO DO SERVIÇO DE PRONTO ATENDIMENTO DE UM HOSPITAL PÚBLICO POR ADULTOS
}

\section{IMPROPER USE OF THE ADULT EMERGENCY SERVICE OF A PUBLIC HOSPITAL BY ADULTS}

\section{USO INCORRECTO DEL SERVICIO DE EMERGENCIAS DE UN HOSPITAL PÚBLICO POR PARTE DE LOS ADULTOS}

\author{
Glaci Aparecida Vigilato ${ }^{1}$, Camila Mucuta da Silva ${ }^{2}$, Luana Vieira Coelho Ferreira ${ }^{3}$, Priscila \\ Aguiar Mendes ${ }^{4}$, Leandro Felipe Mufato ${ }^{5}$, Ana Carolina Macri Gaspar Vendramini ${ }^{6}$
}

Como citar esse artigo: Vigilato GA, Silva CM, Ferreira LVC, Mendes PA, Mufato LF, Vendramini ACMG. Uso inadequado do serviço de pronto atendimento de um hospital público por adultos. Rev Enferm Atenção Saúde [Internet]. 2021 [acesso em:_]; 10(3):e202135. DOI: https://doi.org/ 10.18554/reas.v10i3.4442

\begin{abstract}
RESUMO
OBJETIVO: Identificar o perfil de pacientes adultos atendidos em um pronto atendimento de um hospital público, as características do atendimento e a prevalência do uso inadequado deste serviço. MÉTODO: Estudo transversal, com dados de pesquisa matricial realizada no pronto atendimento de um hospital público no interior de Mato Grosso, com amostra de 538 indivíduos. Foram incluídas informações referentes aos usuários com idade entre 18 a 59 anos e realizada análise descritiva. RESULTADOS: A maioria dos usuários é do sexo feminino, com idade entre 18 e 27 anos, baixa escolaridade e renda familiar. A maior procura foi às terças feiras no período vespertino por sintomas relacionados a doenças infecciosas. A prevalência do uso inadequado do serviço foi de 52,9\%. CONCLUSÃO: Os pacientes que buscam pelo serviço são adultos jovens, mulheres, de baixa renda e escolaridade. Os motivos que tem levado os pacientes a procurar pelo serviço de pronto atendimento são inadequados. Descritores: Emergências; Serviço Hospitalar de Emergência; Sistema Único de Saúde
\end{abstract}

\footnotetext{
${ }^{1}$ Universidade do Estado de Mato Grosso. Enfermeira. Graduação em enfermagem pela Universidade do Estado de Mato Grosso, campus de Tangará da Serra, MT.

${ }^{2}$ Universidade Do Estado de Mato Grosso - campus Tangará da serra, MT. Enfermeira. Mestre em enfermagem. Professora Assistente do curso de graduação em enfermagem da Universidade do Estado de Mato Grosso, campus de Tangará da Serra, MT.

${ }^{3}$ Universidade Do Estado de Mato Grosso - campus Tangará da serra, MT. Enfermeira. Professora Auxiliar do curso de graduação em enfermagem da Universidade do Estado de Mato Grosso, campus de Tangará da Serra, MT.

${ }^{4}$ Universidade Do Estado de Mato Grosso - campus Tangará da serra, MT. Enfermeira. Mestre em enfermagem. Professora Interina do curso de graduação em enfermagem da Universidade do Estado de Mato Grosso, campus de Tangará da Serra, MT.

${ }^{5}$ Universidade Do Estado de Mato Grosso - campus Tangará da serra, MT. Enfermeiro. Doutor em enfermagem. Professor Adjunto do curso de graduação em enfermagem da Universidade do Estado de Mato Grosso, campus de Tangará da Serra, MT.

${ }^{6}$ Universidade Do Estado de Mato Grosso - campus Tangará da serra, MT. Enfermeira. Mestre em enfermagem. Professora Assistente do curso de graduação em enfermagem da Universidade do Estado de Mato Grosso, campus de Tangará da Serra, MT.
} 


\begin{abstract}
OBJECTIVE: To identify the profile of adult patients seen in an emergency room at a public hospital, the characteristics of the service and the prevalence of inappropriate use of this service. METHOD: Cross-sectional study with data from matrix research carried out in the emergency department of a public hospital in the interior of Mato Grosso, with a sample of 538 individuals. Information regarding users aged 18 to 59 years was included and a descriptive analysis was performed. RESULTS: Most users are female, aged between 18 and 27 years old, with low education and family income. The greatest demand was on Tuesdays in the afternoon for symptoms related to infectious diseases. The prevalence of inappropriate use of the service was 52.9\%. CONCLUSION: Patients seeking the service are young adults, women, with low income and education. The reasons that have led patients to seek emergency care are inadequate.
\end{abstract}

Descriptors: Emergencies; Emergency Service, Hospital; Unified Health System

\title{
RESUMÉN
}

OBJETIVO: Identificar el perfil de pacientes adultos atendidos en un departamento de emergencias de un hospital público, las características del servicio y la prevalencia del uso inapropiado de este servicio. MÉTODO: Estudio transversal con datos de investigación matricial realizada en el departamento de emergencias de un hospital público en el interior de Mato Grosso, con una muestra de 538 individuos. Se incluyó información sobre usuarios de 18 a 59 años y se realizó un análisis descriptivo. RESULTADOS: La mayoría de los usuarios son mujeres, con edades comprendidas entre 18 y 27 años, con baja educación e ingresos familiares. La mayor demanda fue los martes por la tarde por síntomas relacionados con enfermedades infecciosas. La prevalencia del uso inapropiado del servicio fue del 52,9\%. CONCLUSIÓN: Los pacientes que buscan el servicio son adultos jóvenes, mujeres, con bajos ingresos y educación. Las razones que han llevado a los pacientes a buscar atención de emergencia son inadecuadas.

Descriptores: Urgencias Medicas; Servicio de Urgencia en Hospital; Sistema Único de Salud.

\section{INTRODUÇÃO}

$\mathrm{O}$ atendimento de casos de urgência ou emergência no Sistema Único de Saúde (SUS) brasileiro ocorrem em unidades de assistência de complexidade intermediária, como as Unidades de Pronto Atendimento (UPA), ou em unidades de alta complexidade, como Pronto Atendimento (PA) dos hospitais. Esses serviços de urgência e emergência são caracterizados pelo atendimento a pacientes que estejam em risco iminente de morte, ou requeiram atendimento imediato, como pacientes acidentados, com suspeitas de infartos, dores agudas, fraturas e outras intercorrências de saúde que afetam diretamente os sinais vitais. ${ }^{1}$

Emergências em saúde podem ser definidas como situações nas quais o atendimento não pode ser protelado, devendo ser imediato. Já as urgências são situações em que o atendimento pode ser prestado com um tempo maior de espera, porém não superior a duas horas. ${ }^{1}$ 
A assistência em saúde nos casos de urgência e emergência deve ser de forma resolutiva, com qualidade, afim de manter o paciente estabilizado. Primeiro se realiza uma verificação diagnóstica para então, averiguar a necessidade de encaminhá-lo a serviços de atendimento de maiores complexidades. $^{2}$

No entanto, nas UPA e PA, geralmente ocorrem atendimentos que não são casos de urgência ou emergência. Este fato resulta em um alto índice de uso inadequado do serviço, pois grande parte dos atendimentos nas unidades de pronto atendimento hospitalar são destinados a usuários da rede básica de saúde, ou seja, pacientes com intercorrências que poderiam ser atendidas na atenção primária por meio de consultas médicas com hora marcada e ou livre demanda. ${ }^{3}$

O uso inadequado dos serviços de saúde é considerado quando situações não urgentes são atendidas em unidades de urgência e emergência, ou seja, casos que poderiam ter sua resolutividade em unidades ambulatoriais de média ou baixa complexidade. ${ }^{3}$

O serviço hospitalar de PA configura-se como a principal porta de entrada ao sistema de saúde em muitos casos de procura por assistência, com isso a maior parte destes atendimentos são decorrentes de problemas simples que poderiam ser resolvidos em serviços de atenção básica ou especializados ou, ainda, em serviços de urgência de menor complexidade. Os atendimentos a pacientes que deveriam ser realizados em ambulatório constituem uma causa importante no que se refere às filas e superlotação nas unidades de PA e possivelmente, revela uma distribuição desigual da oferta de serviços, não apenas do ponto de vista quantitativo, mas também, qualitativo, na atenção básica, na especializada e também na área hospitalar. ${ }^{3-4}$

Estudos apontam que entre os pacientes que procuram os serviços de PA e que não são casos de urgências ou emergências há predominância de usuários com um perfil jovem, sendo a maioria sexo feminino, que procuraram a unidade de pronto atendimento por demanda espontânea e por problemas agudos de baixa gravidade (resfriado comum, amigdalite, infecção das vias aéreas superiores, infecção do trato urinário e gastroenterite aguda) que poderiam ser resolvidos em Unidades Básicas de Saúde (UBS). ${ }^{5-7}$

\section{A procura por atendimento em PA} pode ser influenciada por condições sociais, epidemiológicas, organização do sistema de saúde e insuficiência na rede de serviços disponíveis para a população, tornando a unidade de emergência o acesso mais rápido a serviços com maior 
tecnologia e resolutividade. ${ }^{8}$ Entretanto muitos fatores podem contribuir para a superlotação dos serviços de urgência e emergência, como o comodismo de usuários que moram em regiões próximas a essas unidades de saúde, falta de informação aos usuários sobre qual unidade de saúde procurar em casos não urgentes, falha na orientação por profissionais da saúde. Como consequência disso ocorre a superlotação e reduz a capacidade de prestar atendimento de qualidade e em tempo hábil. ${ }^{6}$

Neste sentido, a determinação do uso inadequado dos serviços em uma unidade hospitalar de PA, bem como a classificação do perfil dos usuários que mais procuram este serviço permite que sejam elaboradas e aplicadas estratégias que favoreçam o fluxo de atendimento nestas unidades promovendo mais eficiência e qualidade no serviço prestado. Torna-se importante então, identificar a prevalência do uso inadequado dos serviços de pronto atendimento.

O objetivo deste estudo é identificar o perfil dos pacientes adultos atendidos em um PA de um hospital público, as características do atendimento e a prevalência do uso inadequado deste serviço.

\section{MÉTODO}

Estudo transversal, realizado a partir de uma pesquisa matricial desenvolvida com 538 usuários de um serviço de pronto atendimento de um hospital público, em um município de médio porte do estado de Mato Grosso, em 2016. A partir das entrevistas foi construído um banco de dados, com variáveis referentes ao perfil sociodemográfico, atendimento no hospital e o atendimento na atenção básica.

Foram incluídos neste estudo dados referentes aos usuários com idade de 18 a 59 anos, totalizando uma amostra de 308 participantes, coletados no banco de dados da pesquisa supracitada. As variáveis incluídas para análise foram relacionadas as características sociodemográficas, período do atendimento, motivos da procura pelo serviço de pronto atendimento.

As variáveis sociodemográficas analisadas neste estudo foram: sexo, faixa etária, escolaridade e renda. Já as variáveis das características do atendimento do PA foram motivo que buscou o PA, dia da semana em que procurou o serviço, período do dia, se era a primeira vez, quantas vezes procurou o serviço no último mês e nos últimos seis meses, tempo de espera para a triagem e classificação de risco que recebeu. 
Para classificar a variável uso inadequado do serviço de pronto atendimento foi realizada uma categorização da variável "motivos que levaram os pacientes a procurar o serviço de pronto atendimento", considerado como aqueles motivos ou sinais e sintomas que não se caracterizaram como casos de urgência/emergência. Considerou-se como motivos ou sinais que caracterizem uma urgência/emergência: ter sofrido um acidente (por causas externas); sintomas que sugerem problemas vitais (dor torácica, dor abdominal aguda, dispneia grave- relacionadas a problemas cardiovasculares e respiratórios); quadro conhecido pelo paciente que necessite de hospitalização (problemas obstétricos); encaminhamento médico pela UBS; situações que necessite de atendimento médico rápido.
A análise dos dados foi descritiva com auxílio do programa estatístico e os resultados foram apresentados por meio de tabelas com frequências absolutas e relativas.

O projeto matricial foi aprovado pelo Comitê de Ética em Pesquisa com Seres Humanos da Universidade do Estado de Mato Grosso, com parecer consubstanciado 1.328.397/ 2015.

\section{RESULTADOS}

Dentre os 308 usuários adultos que procuraram o serviço PA a maioria é do sexo feminino $(58,8 \%)$ e a maior parte possui idade entre 18 e 27 anos (38,6\%), ensino médio completo $(27,6 \%)$ e renda familiar mensal inferior a dois saláriosmínimos (41,2\%) (Tabela 1). 
Tabela 1 - Distribuição dos usuários adultos atendidos no Pronto Atendimento do hospital público segundo características sociodemográficas. Tangará da Serra, Mato Grosso, 2016

\begin{tabular}{|c|c|c|}
\hline Variáveis & Frequência(n) & Porcentagem(\%) \\
\hline \multicolumn{3}{|l|}{ Sexo } \\
\hline Feminino & 181 & 058,8 \\
\hline Masculino & 119 & 038,6 \\
\hline Não respondeu & 008 & 002,6 \\
\hline \multicolumn{3}{|l|}{ Faixa etária } \\
\hline 18-27 anos & 119 & 038,6 \\
\hline 28-37 anos & 093 & 030,2 \\
\hline $38-47$ anos & 056 & 018,2 \\
\hline $48-59$ anos & 040 & 013,0 \\
\hline \multicolumn{3}{|l|}{ Escolaridade } \\
\hline Analfabeto & 006 & 001,9 \\
\hline Sabe ler e escrever & 005 & 001,6 \\
\hline Fundamental incompleto & 077 & 025,0 \\
\hline Fundamental Completo & 034 & 011,1 \\
\hline Ensino Médio Incompleto & 048 & 015,6 \\
\hline Ensino Médio Completo & 085 & 027,6 \\
\hline Ensino Superior Incompleto & 036 & 011,7 \\
\hline Ensino Superior completo & 012 & 003,9 \\
\hline Não sabe/não respondeu & 005 & 001,6 \\
\hline \multicolumn{3}{|l|}{ Renda } \\
\hline <2 Salários Mínimos & 127 & 041,2 \\
\hline <1 Salários Mínimo & 074 & 024,0 \\
\hline <3 Salários Mínimo & 072 & 023,4 \\
\hline >4 Salários Mínimo & 011 & 003,6 \\
\hline Não sabe/Não respondeu & 024 & 007,8 \\
\hline TOTAL & 308 & 100,0 \\
\hline
\end{tabular}

Fonte: Próprio autor

A maior parte dos pacientes procurou por atendimento no PA às terças-feiras $(23,1 \%)$ e no período vespertino $(43,5 \%)$. Quanto ao tempo de espera pelo atendimento, a maioria $(61,7 \%)$ relatou ter aguardado menos de 15 minutos para a triagem e não souberam informar a classificação de risco para o atendimento $(81,2 \%)$ (Tabela 2).
A maior parte dos usuários procurou o PA por sintomas relacionados a doenças infecciosas $(23,7 \%)$. A maioria $(83,4 \%)$ relatou não ser a primeira vez que procurou o serviço do $\mathrm{PA}, 65,9 \%$ não buscaram por atendimento no último mês e $39,0 \%$ nos últimos seis meses $(39,0 \%)$. A prevalência de uso inadequado do serviço de PA foi de 52,9\% (Tabela 2). 
Tabela 2 - Distribuição dos usuários adultos atendidos no Pronto Atendimento de um hospital público segundo uso do serviço. Tangará da Serra, Mato Grosso, 2016

\begin{tabular}{|c|c|c|}
\hline Variáveis & Frequência (n) & Porcentagem (\%) \\
\hline \multicolumn{3}{|l|}{ Dia da semana de procura do PA } \\
\hline Segunda-feira & 48 & 15,6 \\
\hline Terça-feira & 71 & 23,1 \\
\hline Quarta-feira & 64 & 20,8 \\
\hline Quinta-feira & 36 & 11,7 \\
\hline Sexta-feira & 35 & 11,4 \\
\hline Sábado & 27 & 08,8 \\
\hline Domingo & 25 & 08,1 \\
\hline Não se aplica & 02 & $\mathbf{0 0 , 5}$ \\
\hline \multicolumn{3}{|l|}{ Período da procura pelo PA } \\
\hline Matutino & 81 & 26,3 \\
\hline Vespertino & 134 & 43,5 \\
\hline Noturno & 89 & 28,9 \\
\hline Não se aplica & 04 & 01,3 \\
\hline \multicolumn{3}{|l|}{ Primeira vez que procura o $\mathbf{P A}$} \\
\hline Não & 257 & 083,4 \\
\hline Sim & 051 & 016,6 \\
\hline \multicolumn{3}{|l|}{$\begin{array}{l}\text { Quantidade de vezes procurou o PA no último } \\
\text { mês }\end{array}$} \\
\hline Nenhuma & 203 & 65,9 \\
\hline Uma & 049 & 15,9 \\
\hline Duas & 031 & 10,1 \\
\hline Três & 013 & 4,2 \\
\hline Quatro & 003 & 01,0 \\
\hline Cinco & 007 & 02,3 \\
\hline Não souberam informar & 002 & 00,6 \\
\hline \multicolumn{3}{|l|}{$\begin{array}{l}\text { Quantidade de vezes que procurou o PA nos } \\
\text { últimos seis meses }\end{array}$} \\
\hline Nenhuma & 120 & 039,0 \\
\hline Uma & 063 & 020,5 \\
\hline Duas & 053 & 017,2 \\
\hline Três & 029 & 009,4 \\
\hline Quatro & 010 & 003,2 \\
\hline Cinco & 009 & 002,9 \\
\hline Mais de seis & 017 & 005,5 \\
\hline Não souberam informar & 007 & 002,3 \\
\hline \multicolumn{3}{|l|}{ Tempo de espera para triagem } \\
\hline$<15$ MIN & 190 & 061,7 \\
\hline$<30 \mathrm{MIN}$ & 062 & 020,1 \\
\hline$<1 \mathrm{HORA}$ & 037 & 012,0 \\
\hline$>1 \mathrm{HORA}$ & 013 & 004,2 \\
\hline Não souberam informar & 006 & 002,0 \\
\hline \multicolumn{3}{|l|}{ Classificação de risco } \\
\hline Não souberam informar & 250 & 081,2 \\
\hline Verde & 023 & 007,5 \\
\hline Amarelo & 015 & 004,9 \\
\hline Azul & 013 & 04,2 \\
\hline Vermelho & 007 & 002,2 \\
\hline \multicolumn{3}{|l|}{ Motivo da procura do hospital } \\
\hline Sintomas relacionados à doenças infecciosas; & 073 & 023,7 \\
\hline Sintomas relacionados à doenças respiratórias & 023 & 007,5 \\
\hline $\begin{array}{llll}\text { Sintomas relacionados } & \text { a } & \text { problemas } \\
\text { gastrointestinais; }\end{array}$ & 038 & 012,3 \\
\hline Sintomas relacionados à doenças cardiovasculares & 021 & 006,8 \\
\hline Sintomas relacionados a problemas renais/ & 016 & 005,2 \\
\hline
\end{tabular}

Rev Enferm Atenção Saúde [Online]. Out/Dez 2021; 10(3):e202135 


\begin{tabular}{lll}
\hline geniturinários; & & \\
\hline Causas externas & 039 & 012,7 \\
\hline Alergias/inflamações de unhas/olhos/dentes. & 038 & 012,3 \\
\hline Algia músculo esquelética & 035 & 011,5 \\
\hline Gravidez, parto e puerpério; & 014 & 004,5 \\
\hline Encaminhado pela UBS & 005 & 001,6 \\
\hline Sintomas de transtorno mentais & 003 & 001,0 \\
\hline Neoplasias/ Alterações de Pele & 002 & 000,6 \\
\hline Não souberam informar & 001 & 000,3 \\
\hline Uso inadequado do serviço & & \\
\hline Sim & 163 & 052,9 \\
\hline Não & 145 & 047,1 \\
\hline TOTAL & $\mathbf{3 0 8}$ & $\mathbf{1 0 0 , 0}$
\end{tabular}

\section{DISCUSSÃO}

Os resultados deste estudo evidenciam um perfil sociodemográfico também encontrado em outras pesquisas ${ }^{5,7-}$ ${ }^{8}$, nas quais a maioria dos pacientes que procuram por atendimentos no PA eram do sexo feminino. Historicamente, na visão de senso comum, tem-se, que o homem é um ser forte, que dificilmente adoece, razão pela qual a procura pelos serviços de saúde apresenta predominância feminina. Além disso, geralmente as mulheres buscam mais os serviços de saúde e cuidam mais de sua saúde. ${ }^{9}$

$\mathrm{O}$ perfil de faixa etária de maior procura do serviço de PA foi de usuários com idades entre 18 e 27 anos, bem como outras análises que obtiveram em diferentes regiões do país, os jovens como maior procura entre 19 a 29 anos. ${ }^{7,10}$

É possível perceber que a maioria dos indivíduos se encontra na faixa etária produtiva, o que pode assinalar para dificuldades em obter atendimento nas
UBS ou por motivo de trabalho, fato pelo qual, os mesmos podem estar procurando o serviço de PA. Outra explicação seria o fato de que dentro desta faixa etária, as pessoas estão mais suscetíveis a acidentes de trabalho, de trânsito, doenças agudas e outras causas externas, o que, por conseguinte leva a busca por uma unidade de PA. ${ }^{11}$

Quanto a escolaridade, o ensino fundamental incompleto e médio completo são os mais prevalentes e com porcentagens bem próximas $(24,7 \%$ e $27,6 \%$ ), resultado semelhante a outros estudos, nos quais o ensino fundamental incompleto foi o de maior prevalência. ${ }^{5,12-}$ 13

Com relação a renda familiar, este estudo evidenciou resultados semelhante a outro estudo. ${ }^{13}$ A baixa escolaridade, exibida pelos usuários do serviço PA, aparece na literatura em parceria com o baixo poder aquisitivo da população que faz uso deste serviço. Este dado pode 
expressar a vulnerabilidade social dos mesmos, evidenciando situações que se refletem nas condições de trabalho. Observa-se que o rendimento condiciona os indivíduos às limitações cotidianas, à qualidade e quantidade de bens e aquisição, incluindo vida social e afetando a saúde. Corroborando, assim, para uma maior procura pelos serviços de saúde por aqueles que apresentam menores rendimentos. ${ }^{13}$

Quanto aos dias e períodos de maior procura pelo serviço, constatou -se que os usuários buscaram atendimento durante a semana e em períodos diurnos, divergindo a expectativa de que seriam os finais de semana e períodos noturnos, pois nestes dias e horários as UBS e ou USF (Unidade de Saúde da Família) não estariam em funcionamento. Outros estudos também encontraram resultados semelhantes. , $^{8,10,14}$ Neste estudo, terça e quarta feira se mostraram como os dias em que a procura do PA se mostrou mais frequente, sendo sábado e domingo os percentuais mais baixos. Esse dado contraria parcialmente a interpretação de que o horário de trabalho no mesmo horário de atendimento em unidades básicas seria um dos motivos para a busca de atendimento no PA, causando uma superlotação nos períodos noturnos ou em finais de semana devido ao fechamento das UBS. Contudo, os períodos vespertinos de terças e quartas são horários em que as unidades básicas de saúde estão abertas para atendimento.

Diante destes resultados, nota-se que a população estudada conserva uma característica hospitalocêntrica, uma vez que no horário de atendimento nas UBS, estes procuram atendimento nas unidades de PA. Este fato pode ser decorrente da demora no agendamento de consultas nas UBS, os horários de atendimento nos mesmos turnos de trabalho dos usuários, além de que no hospital, a aquisição de medicações é mais facilitada, há melhores recursos tecnológicos, os exames são realizados com maior rapidez, além de oferecer também o atendimento especializado. $^{7,15}$

A maioria dos entrevistados afirmaram não ser a primeira vez que procuraram o serviço do PA. No entanto, uma grande parte dos usuários $(32,8 \%)$ relatou que procurou pelo serviço entre duas ou mais de seis vezes nos últimos seis meses, corroborando com outras pesquisas que encontraram, uma média $16,4 \%$ e $37,83 \%$ de indivíduos que procuraram o serviço quatro vezes ou mais durante o ano. ${ }^{13-14}$ Ademais, como no momento das entrevistas eles estavam aguardando ou saindo do atendimento no PA, o fato de não ser a primeira vez que procuram no último mês e nos últimos seis meses, mostra que são sujeitos que reconhecem o serviço como local para a busca inicial de 
cuidados, reforçando a crença que a população tem de que o hospital é mais resolutivo como porta de entrada nos serviços de saúde.

Em relação ao tempo de espera para realização da triagem, em um estudo sobre tempo empregado na classificação de risco e prioridade para atendimento em uma emergência realizado no município de Porto Alegre, Rio Grande do Sul, também encontrou o mesmo tempo de espera deste estudo, menor que quinze minutos. ${ }^{16}$ Cabe ressaltar que o tempo de espera menor que 15 minutos demonstra a eficiência no atendimento e classificação de risco.

Para a classificação de risco de pacientes, os serviços de PA utilizam o Sistema Manchester, no qual se classifica o grau de gravidade e tempo destinado a possível espera por meio de cores, em que a cor vermelha determina condição de emergência, a laranja, muita urgência, já a verde e azul seriam casos de pouca urgência em que o atendimento pode ocorrer após maior tempo, até 120 minutos. $^{17}$

Entretanto, em relação a classificação de risco, assim como neste estudo, em uma pesquisa realizada em uma UPA na cidade de Campo Grande, Mato Grosso do Sul, descobriu-se que os pacientes atendidos não sabiam qual classificação de risco receberam. ${ }^{18}$ Logo, apesar da eficiência na espera para ser triado, os sujeitos entrevistados, em sua maioria, não receberam informações sobre sua classificação.

Este resultado é importante uma vez que a informação não está sendo transmitida aos pacientes. A realização da classificação de risco requer do enfermeiro avaliação clínica rápida e habilidades que são influenciados pelo conhecimento clínico científico e pela experiência profissional. No entanto observa-se que há um grande predomínio de profissionais de nível técnico, na realização da classificação de risco em UBS e UPA, mesmo não sendo capacitados para este procedimento. ${ }^{19}$ Fato este que pode contribuir para elevada demanda de sobrelotação dos PA, desqualificação do atendimento ou troca de informações entre profissionais e pacientes.

Além disso, o fato que os pacientes desconhecem a classificação de risco recebida contribui para que o mesmo não compreenda o motivo da espera do atendimento, ou até mesmo a indicação de assistência no serviço de urgência/emergência ou se deveria ser atendido na atenção básica, contribuindo para que ele retorne ao serviço de forma inadequada.

Quanto ao motivo da procura pelo serviço o que mais aparece são sintomas relacionados a doenças infeciosas. Um estudo realizado no PA de Santa Maria, 
Rio Grande do Sul, apresentou como motivo de maior procura a febre, que por sua vez, se caracteriza como um dos sintomas relacionados a doenças infecciosas. ${ }^{4}$ Tais achados são semelhantes a outros estudos que também tiveram como maior procura motivos de febre, seguidos de distúrbios gastrointestinais $\mathrm{e}$ respiratórios. ${ }^{3,7}$

Por outro lado, estudos encontraram como maior causa de procura do PA doenças do aparelho circulatório, em que a principal patologia foi a hipertensão ${ }^{12} \mathrm{e} \mathrm{em}$ outros, dor aguda $(26,4 \%)$ seguida por problemas respiratórios $(14,3 \%){ }^{10}$

Cabe ressaltar que, no período de realização desta pesquisa, a incidência de casos de ZIKA em todo o país era alto, coincidindo com os suspeitos de dengue e influenza $\mathrm{H} 1 \mathrm{~N} 1$, o que poderia ter corroborado para o resultado encontrado.

A adequação do uso do serviço de PA tem sido estudada em todo país. Em concordância com os achados deste estudo, a maioria tem encontrado como resultado uma alta prevalência de uso inadequado. Uma pesquisa descobriu uma porcentagem de $58,9 \%$ de uso inadequado dos atendimentos investigados, e observaram um alto índice de consultas que evoluíram para alta após o atendimento $(78,7 \%){ }^{14}$

Foi constatado em um estudo que o índice de pacientes que receberam alta hospitalar no SE (Serviço de Emergência) de um hospital de São Paulo capital foi responsável por $94,5 \%$ dos atendimentos na triagem e que a maioria dos pacientes foram classificados como baixa prioridade. $^{20}$ Para os autores, estes resultados caracterizam o uso inapropriado do serviço, uma vez que grande parte dos pacientes que procuraram o SE poderia ser atendida em serviços de menor complexidade.

A alta demanda do serviço de PA advém de usuários que poderiam ser atendidos em unidades de menor complexidade, neste sentido é observado uma necessidade de atividades educativas para a população, a fim de esclarecer sobre a hierarquização e competência de cada unidade da rede assistencial. Medidas como estas podem colaborar para amortizar valores e acrescer a eficácia nos serviços de PA.

A utilização do PA por usuários que não apresentavam situação de urgência ou emergência, caracterizando uso inadequado da UPA, se deu na maioria dos casos, por demanda espontânea, sem nenhuma consulta ou encaminhamento prévio. Fato este que caracteriza uma necessidade de se pensar em estratégias que viabilize aos usuários uma resolução para problemas de saúde não urgentes, nas UBS, em bairros próximos as suas residências e em horários que possibilitem a procura pelo atendimento. 
Muitos usuários buscam o PA por considerar um serviço mais rápido e de resolução efetiva, porém isso traz filas e superlotação. Com isso, há também, a necessidade de se desenvolver ações educativas, visando orientar a população sobre as características de atendimento de cada serviço de saúde.

Os conhecimentos destes resultados poderão nortear intervenções para diminuir o índice de superlotação no atendimento do serviço de emergência e contribuir para adequações no sistema de atendimento da atenção básica, tornando $\mathrm{o}$ atendimento destas unidades mais rápido e efetivo.

Este estudo possui limitações por ser transversal, com amostragem não probabilística, uma vez que, seus resultados são aplicáveis somente a população estudada. No entanto, pode contribuir para o levantamento de informações do atendimento a fim de orientar intervenções e planejamento de ações para melhorar o uso do serviço de PA pela população.

\section{CONCLUSÃO}

O serviço de PA do hospital municipal é utilizado, na maioria dos casos, por pacientes não urgentes, com uma maior porcentagem de mulheres jovens, baixa escolaridade e renda, que procuram o serviço em dias úteis e em períodos comerciais. Os principais sintomas que levaram à procura do serviço no período do estudo foram por sintomas relacionados a doenças infecciosas.

$\mathrm{O}$ uso de um serviço PA, quando inadequado, pode gerar custos desnecessários ao sistema de saúde, além de um tratamento sem continuidade para os pacientes que necessitam de cuidados contínuos.

Ademais, a qualidade da triagem, com o esclarecimento aos pacientes sobre os protocolos utilizados se mostrou frágil nesta pesquisa, com atendimento rápido, porém desinformado aos usuários dos serviços, assunto sobre o qual pesquisas futuras poderão realizar maiores investigações. Nota-se com isso a necessidade e a importância no desenvolvimento de outros estudos como este que envolvam a verificação de associação entre as variáveis sociodemográficas e os motivos da procura pelo serviço de PA

Este estudo traz achados que servem como subsídios para o planejamento dos gestores municipais no que se refere à organização da rede, elaboração e planejamento de ações e políticas públicas, que terão como objetivo diminuir a superlotação da unidade de PA. Dessa forma, contribui com a possibilidade de os usuários procurarem os serviços de saúde conforme as suas necessidades. 


\section{REFERÊNCIAS}

1. Ministério da Saúde (Brasil). Portaria ${ }^{\circ}$ 354, de 10 de março de 2014. Publica a proposta de Projeto de Resolução Boas Práticas para Organização e Funcionamento de Serviços de Urgência e Emergência (SUS) [Internet]. Brasíllia, DF: Ministério da Saúde; 2014 [citado em 29 jul 2017]. Disponível em: http://bvsms.saude.gov.br/bvs/saudelegis/g m/2014/prt0354_10_03_2014.html 2. Ministério da Saúde (Brasil). Portaria $n^{\circ}$ 10 de 3 de janeiro de 2017. Redefine as diretrizes de modelo assistencial e financiamento de UPA 24h de Pronto Atendimento como Componente da Rede de Atenção às Urgências, no âmbito do Sistema Único de Saúde (SUS)[Internet]. Diário Oficial da União, Brasília, DF, 04 jan 2017 [citado em 29 jul 2017]; Seção 1, (3):34. Disponível em:

https://www.in.gov.br/materia//asset_publisher/Kujrw0TZC2Mb/content/i d/20574215/do1-2017-01-04-portaria-n10-de-3-de-janeiro-de-2017-20574152 3. Camerro A, Alves EC, Camerro NMMS, Nogueira LDP. Perfil do atendimento de serviços de urgência e emergência. Rev Fafibe On-Line [Internet]. 2015 [citado em $14 \mathrm{dez}$ 2021]; 8(1):515-24. Disponível em: https://www.unifafibe.com.br/revistasonline/ar quivos/revistafafibeonline/sumario/36/1011201 5195658.pdf

4. Freire AB, Fernandes DL, Moro JS, Kneipp MM, Cardoso CM, Lima SBS. Serviços de urgência e emergência: quais os motivos que levam o usuário aos pronto-atendimentos? Saúde (Santa Maria) [Internet]. jan/jul 2015 [citado em $14 \mathrm{dez}$ 2021]; 41(1):195-200. Disponível em: https://www.researchgate.net/publication/2 81942553_SERVICOS_DE_URGENCIA_ E_EMERGENCIA_QUAIS_OS_MOTIV OS_QUE_LEVAM_O_USUARIO_AOS_ PRONTO-ATENDIMENTOS

5. Silva PL, Paiva L, Faria VB, Ohl RIB, Chavaglian SRR. Acolhimento com classificação de risco do serviço de prontosocorro adulto: satisfação do usuário. Rev Esc Enferm USP. [Internet]. 2016 [citado em 15 dez 2021]; 50(3):427-33. Disponível em:

https://www.scielo.br/j/reeusp/a/DCH4PfP Qs9z4jSgRxgL8Tjr/?format=pdf\&lang=pt \#: :text=Verificaram\%2Dse\%20altos\%20 $\%$ C3\%ADndices\%20de,Socorro\%20Adult o\%20\%2D\%20HC\%2FUFTM

6. Feijó VBER, Junior LC, Souza RKT, Dias AO. Análise da demanda atendida em unidade de urgência com classificação de risco. Saúde Debate [Internet]. jul/set 2015 [citado em $15 \mathrm{dez}$ 2021]; 39(106):

627-36. Disponível em:

https://www.scielo.br/j/sdeb/a/9rrjMmGS8 Hxdv4ZRVDFPcTn/?format=pdf\&lang=pt 7. Oliveski CC, Santos LE, Marco VR, Lorenzoni AMC, Bonfada MS, Silva LAA. Perfil clínico de usuários de um serviço de emergência. Revista Espaço Ciência \& Saúde [Internet]. dez 2017 [citado em 15 dez 2021]; 5(2):45-56. Disponível em: https://revistaeletronica.unicruz.edu.br/inde x.php/saude/article/view/115/55

8. Acosta AM, Lima MADS. Usuários frequentes do serviço de emergência: fatores associados e motivos de busca por atendimento. Rev Latinoam Enferm. [Internet]. fev/abr 2015 [citado em $15 \mathrm{dez}$ 2021]; 23(2):337-44. Disponível em: https://www.scielo.br/j/rlae/a/MFdMTTGR hG35Gp3YtrkVm6M/?format=pdf\&lang= $\mathrm{pt}$

09. Levorato CD, Mello LM, Silva AS, Nunes AA. Fatores associados à procura por serviços de saúde numa perspectiva relacional de gênero. Ciênc Saúde Colet. [Internet]. abr 2014 [citado em $15 \mathrm{dez} 2021$ ]; 19(4):1263-74. Disponível em: https://www.scielo.br/j/csc/a/8cp6H8fy9rS pQvGG3WcYXKB/?format=pdf\&lang=pt 10. Garcia VM, Reis RK. Perfil de usuários atendidos em uma unidade não hospitalar de urgência. Rev Bras Enferm. [Internet]. mar/abr 2014 [citado em $15 \mathrm{dez}$ 2021]; 67(2):261-7. Disponível em: https://www.scielo.br/j/reben/a/jxrzvyjSYv h3jFRwSQwXQdK/?format=pdf\&lang=pt 11. Rodrigues AIG, Korinfsky JP, Santos ADB, Oliveira ANS, Almeida LR, Moura LA. Perfil dos usuários atendidos no 
serviço de emergência em um hospital universitário em Pernambuco. Rev Baiana Saúde Pública [Internet]. jan/mar 2015 [citado em 15 dez 2021]; 39(1):13-24. Disponível em:

https://rbsp.sesab.ba.gov.br/index.php/rbsp /article/view/1690/pdf_604

12. Coelho MF, Goulart BF, Chaves LDP. Urgências clínicas: perfil de atendimentos hospitalares. Rev Rene (Online) [Internet]. 2013 [citado em 15 dez 2021]; 14(1):50-9.

Disponível em:

http://periodicos.ufc.br/rene/article/view/3 $325 / 2563$

13. Carvalho TP, Okuno MFP, Campanharo CRV, Lopes MCBT, Batista REA. Conhecimento dos pacientes sobre a prescrição medicamentosa no serviço de emergência. Rev Bras Enferm. [Internet]. mar/abr 2018 [citado em $15 \mathrm{dez}$ 2021]; 71(2):329-35. Disponível em: https://www.scielo.br/j/reben/a/XJszSTrjm m5CGj7GKY7hMXm/?format=pdf\&lang= en

14. Machado GVC, Oliveira FLP, Barbosa HAL, Giatti L, Bonolo PF. Fatores associados à utilização de um serviço de urgência/emergência, Ouro Preto, 2012. Cad Saúde Colet. (Rio J.) [Internet]. out/dez 2015 [citado em 15 dez 2021]; 23(4):416-24. Disponível em: https://www.scielo.br/j/cadsc/a/CDfXj4SL SFsDDs3TdvpNvFG/?format $=$ pdf\&lang $=p$ $\mathrm{t}$

15. Oliveira GS, Jesus RM, Lima DM, Mendonça IO, Ribeiro HL. Superlotação das urgências e estratégias de gestão de crise: uma revisão de literatura. Cad Grad, Ciênc Hum Soc. [Internet]. out 2017 [citado em 15 dez 2021]; 4(2):115-26. Disponível em:

https://periodicos.set.edu.br/cadernobiologi cas/article/view/4596/2498

16. Anziliero F, Soler BED, Silva BA, Tanccini T, Beghetto MG. Sistema

Manchester: tempo empregado na classificação de risco e prioridade para atendimento em uma emergência. Rev Gaúch Enferm. [Internet]. dez 2016 [citado em 15 dez 2021]; 37(4):e64753.

Disponível em:

https://www.scielo.br/j/rgenf/a/ZPt8CVtgX pftkT7MszL8KtP/?format=pdf\&lang=pt 17. Guedes HM, Almeida AGP, Ferreira FO, Vieira Júnior G, Chianca TCM. Classificação de risco: retrato de população atendida num serviço de urgência brasileiro. Referência (Coimbra) [Internet]. fev/mar 2014 [citado em 25 abr 2018]; 4(1):37-44. Disponível em: http://www.scielo.mec.pt/pdf/ref/vserIVn1/ serIVn1a05.pdf

18. Pícoli RP, Cazola LHO, Maurer NMJS. Usuários de classificação de risco azul em uma unidade de pronto atendimento. Cogit Enferm. [Internet]; jan/mar 2016 [citado em 15 dez 2021]; 21(1):1-7. Disponível em: https://revistas.ufpr.br/cogitare/article/view /43044/27664

19. Godoi VCG, Ganassin GS, Inoue KC, Gil NLM. Acolhimento com classificação de risco: caracterização da demanda em unidade de pronto atendimento. Cogit Enferm. [Internet]; jul/set 2016 [citado em 03 jan 2021]; 21(3):1-8. Disponível em: https://revistas.ufpr.br/cogitare/article/view /44664/pdf

20. Becker JB, Lopes MCBT, Pinto MF, Campanharo CRV, Barbosa DA, Batista REA. Triagem no serviço de emergência: associação entre as suas categorias e os desfechos do paciente. Rev Esc Enferm USP. [Internet]; 2015 [citado em $15 \mathrm{dez}$ 2021]; 49(5):779-85. Disponível em: https://www.scielo.br/j/reeusp/a/Pgbz7YH Pc8WKYryP5xznF4x/?format=pdf\&lang= en

RECEBIDO: $24 / 04 / 2020$

APROVADO: 08/09/2021

PUBLICADO: dez/2021 